\title{
Publisher's Note: Kaon transverse charge density from space- and timelike data
} [Phys. Rev. C 96, 065207 (2017)]

N. A. Mecholsky, Johann Mejia-Ott, M. Carmignotto, T. Horn, G. A. Miller, and I. L. Pegg

(Q) (Received 22 February 2019; published 1 March 2019)

DOI: 10.1103/PhysRevC.99.039901

This paper was published online on 26 December 2017 with a misspelling in the second author's name. The second author's name should read as "Johann Mejia-Ott." The author's name has been corrected as of 13 February 2019. The author's name is incorrect in the printed version of the journal. 